\title{
The optimal compensator for nonlinear phase noise
}

\author{
Keang-Po $\mathrm{Ho}^{\mathrm{a}, \mathrm{b}, *}$ \\ a StrataLight Communications, 2105 S. Bascom Ave. Suite 300, Campbell, CA 95008, USA \\ ${ }^{\mathrm{b}}$ Institute of Communications Engineering, National Taiwan University, Taipei 106, Taiwan \\ Received 25 January 2003; received in revised form 21 March 2003; accepted 8 May 2003
}

\begin{abstract}
The nonlinear phase noise, often called the Gordon-Mollenauer effect, is correlated with the received intensity. The optimal compensator is the minimum mean-square error (MMSE) compensator to estimate the nonlinear phase noise using the received intensity. The MMSE compensator is derived analytically and approximated by subtracting a correction term proportional to the received intensity.
\end{abstract}

(c) 2003 Elsevier Science B.V. All rights reserved.

PACS: 42.65.-k; 05.40.-a; 42.81.Uv

Keywords: Nonlinear phase noise; Fiber nonlinearities; Noise compensation

\section{Introduction}

Gordon and Mollenauer [1] showed that when optical amplifiers are used in lightwave communication systems, the interaction of amplifier noise and fiber Kerr effect causes phase noise, often called the GordonMollenauer effect. Nonlinear phase noise degrades the performance of both phase-shifted keying (PSK) and differential phase-shift keying (DPSK) systems. This class of constant-intensity modulation have renewed attention recently [2-4]. Because the nonlinear phase noise is correlated with the received intensity, the received intensity can be used to correct the nonlinear phase noise [5-7]. The transmission distance can approximately be doubled if the nonlinear phase noise is the dominant impairment [5,7].

In all nonlinear phase noise compensators [5-7], the correction term is proportional to the received intensity, called linear compensator in later parts of this paper. While the all-optical compensator [5] is limited by the available nonlinear phase shifter, the electro-optic implementation [6] and all-electronic implementation [7] can use electronic signal processing to provide arbitrary nonlinear functions. To correct the nonlinear phase noise, a fundamental problem is to find the optimal compensator without the

\footnotetext{
${ }^{*}$ Tel.: +1-408-961-6259; fax: +1-408-626-7100.

E-mail address: kpho@stratalight.com (K.-P. Ho).
} 
constraint of linearity. It is also important to verify whether the linear compensator is close to the optimal compensator.

The optimal compensator should minimize the variance of the residual nonlinear phase noise [5-7]. The optimal compensator is the minimum mean-square error (MMSE) estimator to estimate the nonlinear phase noise using the received intensity. This paper derives the MMSE compensator analytically the first time. The linear compensator derived in [7] can be used to well approximate the nonlinear MMSE compensator.

\section{The minimum mean-square error estimator}

For an $N$-span system, the total nonlinear phase noise is $[1,5,7]$

$$
\phi_{\mathrm{NL}}=\gamma L_{\mathrm{eff}}\left[\left|E_{0}+n_{1}\right|^{2}+\left|E_{0}+n_{1}+n_{2}\right|^{2}+\cdots+\left|E_{0}+n_{1}+\cdots+n_{N}\right|^{2}\right],
$$

where $E_{0}$ is the transmitted signal, $n_{k}, k=1, \ldots, N$, are the optical amplifier noise introduced into the system at the $k$ th fiber span, $n_{k}$ are independent identically distributed (i.i.d.) complex zero-mean circular Gaussian random variables with $E\left\{\left|n_{k}\right|^{2}\right\}=2 \sigma^{2}$, where $\sigma^{2}$ is the noise variance per dimension per span, $\gamma$ is the fiber nonlinear coefficient, and $L_{\text {eff }}$ is the effective length per span. In the nonlinear phase noise of (1), all fiber spans are assumed to be identical with the same launched power, the same fiber length, the same span loss, and using optical amplifiers having the same noise figure. The amplifier noise from the polarization orthogonal to the signal is ignored [1]. Nonlinear phase noise of (1) is induced by self-phase modulation of the amplifier noise having a bandwidth matched to the transmitted signal. Cross-phase modulation induced phase noise is ignored in (1).

The received intensity $\rho_{\mathrm{r}}^{2}$ is used to compensate the nonlinear phase noise of (1), where the received amplitude is

$$
\rho_{\mathrm{r}}=\left|E_{0}+n_{1}+\cdots+n_{N}\right| \text {. }
$$

The received amplitude of (2) ignores the fiber loss of the last fiber span and the required optical amplifier to compensate for it. The actual received electric field is

$$
E_{\mathrm{r}}=\left(E_{0}+n_{1}+\cdots+n_{N}\right) \exp \left(-\mathrm{j} \phi_{\mathrm{NL}}\right)
$$

with nonlinear phase rotation of $\phi_{\mathrm{NL}}$. The nonlinear phase noise of (1) does not change the received amplitude $\rho_{\mathrm{r}}$ of (2). For binary PSK and DPSK, only the real part of the received electric field or the corresponding real differential component is required to detect the transmitted data. Both real and imaginary part of the electric field of $E_{\mathrm{r}}$ (3) can be measured by an optical phase-locked loop for PSK signal [8] and the corresponding differential components can be measured by two interferometers for DPSK signal [3]. The optimal compensator minimizes the phase noise in the received electric field of $E_{\mathrm{r}}$ by minimize the variance of the residual nonlinear phase noise. Using the received intensity $\rho_{\mathrm{r}}^{2}$, the optimal compensator is the MMSE estimator based on the conditional mean

$$
E\left\{\phi_{\mathrm{NL}} \mid \rho_{\mathrm{r}}\right\}
$$

where $E\{\cdot\}$ denotes expectation.

The variance of $\phi_{\mathrm{NL}}-E\left\{\phi_{\mathrm{NL}} \mid \rho_{\mathrm{r}}\right\}$ is minimized using the nonlinear MMSE compensator of (4). The optimal compensator can be implemented using a phase modulator [6] driven by the waveform of (4) or an electrical compensator to calculate $E_{\mathrm{r}} \exp \left[\mathrm{j} E\left\{\phi_{\mathrm{NL}} \mid \rho_{\mathrm{r}}\right\}\right]$ using electronic signal processing [7]. If the received phase is demodulated as $\phi_{\mathrm{r}}=\arg \left(E_{\mathrm{r}}\right)$, the compensator is a combiner of $\phi_{\mathrm{r}}+E\left\{\phi_{\mathrm{NL}} \mid \rho_{\mathrm{r}}\right\}$. There is no difference between $E\left\{\phi_{\mathrm{NL}} \mid \rho_{\mathrm{r}}^{2}\right\}$ and the expectation of (4) because the received amplitude is a positive number $\rho_{\mathrm{r}} \geqslant 0$. Here, we refer the estimator of (4) as a correction by received intensity although the expression of (4) is based on received amplitude. 
Without loss of generality, assume that the transmitted electric field is $E_{0}=A$ as a real value, the results of Appendix A can be used to estimate

$$
E\left\{\left|A+n_{1}+\cdots+n_{k}\right|^{2}|| A+n_{1}+\cdots+n_{N} \mid\right\} .
$$

Compared the estimation of (5) with (A.1) of Appendix A, we have $\xi_{1}=n_{1}+\cdots+n_{k}$ and $\xi_{2}=n_{k+1}+\cdots+n_{N}$ that are zero-mean complex circular Gaussian random variables with variance of $E\left\{\left|\xi_{1}\right|^{2}\right\}=2 k \sigma^{2}$ and $E\left\{\left|\xi_{2}\right|^{2}\right\}=2(N-k) \sigma^{2}$. Using the function of $\varphi\left(\sigma_{1}, \sigma_{2}\right)$ defines in (A.1) of Appendix A, we get

$$
E\left\{\left|A+n_{1}+\cdots+n_{k}\right|^{2} \mid \rho_{\mathrm{r}}\right\}=\varphi(\sqrt{k} \sigma, \sqrt{N-k} \sigma) .
$$

For the estimator of (4), we get

$$
E\left\{\phi_{\mathrm{NL}} \mid \rho_{\mathrm{r}}\right\}=\gamma L_{\mathrm{eff}} \sum_{k=1}^{N} \varphi(\sqrt{k} \sigma, \sqrt{N-k} \sigma) .
$$

Substitute the function of $\varphi\left(\sigma_{1}, \sigma_{2}\right)$ of (A.8) of Appendix A to (7), we get

$$
\begin{aligned}
E\left\{\phi_{\mathrm{NL}} \mid \rho_{\mathrm{r}}\right\}= & \gamma L_{\mathrm{eff}}\left\{\frac{(2 N-1)(N-1)}{6 N} A^{2}+\frac{N^{2}-1}{3} \sigma^{2}+\frac{(2 N+1)(N+1)}{6 N} \rho_{\mathrm{r}}^{2}\right. \\
& \left.+\frac{\left(N^{2}-1\right) A \rho_{\mathrm{r}}}{3 N} \frac{I_{1}\left(A \rho_{\mathrm{r}} /\left(N \sigma^{2}\right)\right)}{I_{0}\left(A \rho_{\mathrm{r}} /\left(N \sigma^{2}\right)\right)}\right\},
\end{aligned}
$$

where $I_{0}(\cdot)$ and $I_{1}(\cdot)$ are the zeroth- and first-order modified Bessel function of the first kind. To derive (8), the following relationships are used:

$$
\begin{aligned}
& \sum_{k=1}^{N} k^{2}=\frac{1}{6} N(2 N+1)(N+1), \\
& \sum_{k=1}^{N}(N-k)^{2}=\frac{1}{6} N(2 N-1)(N-1), \\
& \sum_{k=1}^{N} k^{2}(N-k)=\sum_{k=1}^{N} k(N-k)^{2}=\frac{1}{12} N^{2}\left(N^{2}-1\right), \\
& \sum_{k=1}^{N} k(N-k)=\frac{1}{6} N\left(N^{2}-1\right) .
\end{aligned}
$$

Because $I_{1}(x) / I_{0}(x) \sim x / 2$ for small $x<0.5$, the phase estimator of $(8)$ is a linear function of the received intensity of $\rho_{\mathrm{r}}^{2}$ when the received signal is small. Because $I_{1}(x) / I_{0}(x) \sim 1$ for large $x>10$, the phase estimator of (8) is a quadratic function of the received amplitude of $\rho_{\mathrm{r}}$ when the received signal is large. The interested systems usually have high optical signal-to-noise ratio (SNR) of $\rho_{\mathrm{O}}=A^{2} /\left(2 N \sigma^{2}\right)$ and the received signal has an amplitude around $A$, i.e., $\rho_{\mathrm{r}} \sim A$, the phase estimator of (8) mostly functions as a nonlinear compensator.

For large number of fiber span of $N \gg 1$ and high optical SNR, the phase estimator of (8) is

$$
E\left\{\phi_{\mathrm{NL}} \mid \rho_{\mathrm{r}}\right\}=\frac{\left\langle\phi_{\mathrm{NL}}\right\rangle}{3}\left[1+\frac{\rho_{\mathrm{r}}^{2}}{A^{2}}+\frac{\rho_{\mathrm{r}}}{A} \frac{I_{1}\left(\rho_{\mathrm{O}} A / \rho_{\mathrm{r}}\right)}{I_{0}\left(\rho_{\mathrm{O}} A / \rho_{\mathrm{r}}\right)}\right],
$$


where the mean nonlinear phase noise $\left\langle\phi_{\mathrm{NL}}\right\rangle=N A^{2}+N(N+1) \sigma^{2} \approx N A^{2}$. The estimation of (13) divides the estimation into three parts, the first term is one third the mean nonlinear phase, the second term proportional to received intensity, and third term is the nonlinear term.

\section{Numerical results}

Fig. 1 show the simulated distribution and the decision regions of the received signal with nonlinear phase noise. The received electric field $E_{\mathrm{r}}$ (3) includes the contribution of amplifier noise of $E_{0}+n_{1}+\cdots+n_{N}$. The mean nonlinear phase noise $\left\langle\phi_{\mathrm{NL}}\right\rangle$ are 1 and 2 rad for Figs. 1(a) and 1(b), respectively. The mean nonlinear phase noise of $\left\langle\phi_{\mathrm{NL}}\right\rangle=1 \mathrm{rad}$ corresponds to the limitation estimated by [1]. The mean nonlinear phase noise of $\left\langle\phi_{\mathrm{NL}}\right\rangle=2 \mathrm{rad}$ corresponds to the limitation given by [7] when the variance of nonlinear phase noise is reduced by a factor of four using a linear compensator. The decision boundaries shown as solid line are the MMSE compensator of (8). The dotted lines are the optimal decision boundary using the linear compensator [7].

Fig. 1 are plotted for the case that the optical SNR $\rho_{\mathrm{O}}=18$, corresponding to an error probability of $10^{-9}$ if the amplifier noise is the sole impairment. The number of spans is $N=32$. The transmitted signal is $\pm A$ for binary PSK system, the distribution of Fig. 1 has 5000 points for different noise combinations.

Fig. 1 show that the decision regions given by the MMSE estimator of (8) and that given by [7] has small difference. The decision boundary given by [7] for the case of $\left\langle\phi_{\mathrm{NL}}\right\rangle=1 \mathrm{rad}$ has larger rotation then the MMSE curve given by (8). However, the decision boundary given by [7] for the case of $\left\langle\phi_{\mathrm{NL}}\right\rangle=2 \mathrm{rad}$ has smaller rotation then the MMSE curve given by (8). Further numerical results show that the decision boundary given by [7] and the MMSE curve of (8) almost overlap with each other when $\left\langle\phi_{\mathrm{NL}}\right\rangle=1.5$.

Fig. 2 shows the simulated phase noise standard deviation as a function of mean nonlinear phase noise with and without compensation. For all 5000 points in Fig. 1, the standard deviation of the nonlinear phase noise $\phi_{\mathrm{NL}}$ of (1), denotes as $\sigma_{\phi_{\mathrm{NL}}}$, is calculated. When the nonlinear phase noise is compensated using a linear compensator of $\alpha \rho_{\mathrm{r}}^{2}$ proportional to the received intensity of $\rho_{\mathrm{r}}^{2}$, the standard deviation of the residual nonlinear phase noise of $\phi_{\mathrm{NL}}-\alpha \rho_{\mathrm{r}}^{2}$ is calculated and denotes as $\sigma_{\phi_{\mathrm{NL}}-\alpha \rho_{\mathrm{r}}^{2}}$, where $\alpha$ is the scale factor. When the nonlinear phase noise is compensated using the MMSE compensator $E\left\{\phi_{\mathrm{NL}} \mid \rho_{\mathrm{r}}\right\}$ of (8), the standard deviation of the residual nonlinear phase noise of $\phi_{\mathrm{NL}}-E\left\{\phi_{\mathrm{NL}} \mid \rho_{\mathrm{r}}\right\}$ is calculated and denotes as $\sigma_{\phi_{\mathrm{NL}}-E\left\{\phi_{\mathrm{NL}} \mid \rho_{\mathrm{r}}\right\}}$. Fig. 2 also plots the approximations [7]

$$
\sigma_{\phi_{\mathrm{NL}}} \approx 0.1925\left\langle\phi_{\mathrm{NL}}\right\rangle \text { and } \sigma_{\phi_{\mathrm{NL}}-\alpha \rho_{\mathrm{r}}^{2}} \approx 0.1925\left\langle\phi_{\mathrm{NL}}\right\rangle \text {. }
$$

Fig. 2 shows that there is almost no difference between the standard deviation of the residual nonlinear phase noise of $\sigma_{\phi_{\mathrm{NL}}-\alpha \rho_{\mathrm{r}}^{2}}$ using the linear compensator [7] and the standard deviation of the residual nonlinear

(a)

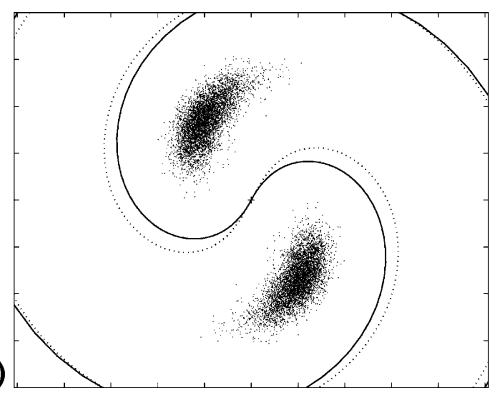

(b)

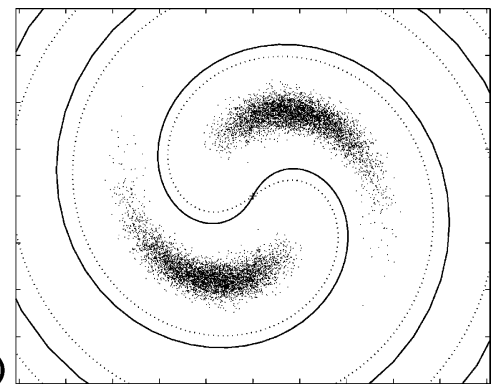

Fig. 1. Simulated distribution and the decision regions of the received signal for mean nonlinear phase noises of (a) $\left\langle\phi_{\mathrm{NL}}\right\rangle=1 \mathrm{rad}$ and (b) $\left\langle\phi_{\mathrm{NL}}\right\rangle=2 \mathrm{rad}$. The decision boundary is given by solid line (8) and dotted line [7]. 


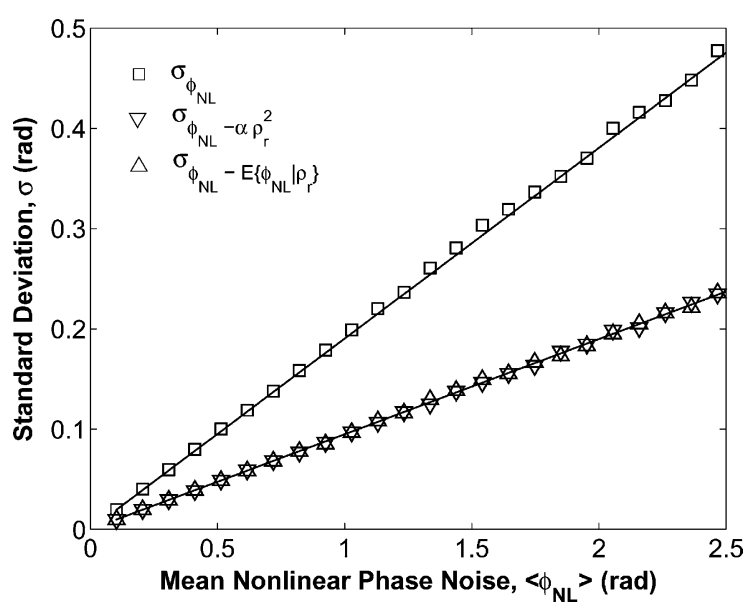

Fig. 2. The standard deviation of nonlinear phase noise with and without compensation. The solid lines are the approximation of (14).

phase noise of $\sigma_{\phi_{\mathrm{NL}}-E\left\{\phi_{\mathrm{NL}} \mid \rho_{\mathrm{r}}\right\}}$ using the MMSE compensator (8). In general, $\sigma_{\phi_{\mathrm{NL}}-E\left\{\phi_{\mathrm{NL}} \mid \rho_{\mathrm{r}}\right\}}$ is smaller than $\sigma_{\phi_{\mathrm{NL}}-\alpha \rho_{\mathrm{r}}^{2}}$ by about only $0.2 \%$. Fig. 2 also show that the approximation of (14) is also very accurate.

\section{Discussion}

While most of the above section is related to PSK systems, as shown in [7], the optimal compensator for DPSK systems follows the same principle. In DPSK system, the differential phase of $\phi_{\mathrm{r}}(t)-\phi_{\mathrm{r}}(t-T)$ is detected, where $T$ is the symbol period. The optimal MMSE compensator should be

$$
E\left\{\phi_{\mathrm{NL}}(t) \mid \rho_{\mathrm{r}}(t)\right\}-E\left\{\phi_{\mathrm{NL}}(t-T) \mid \rho_{\mathrm{r}}(t-T)\right\},
$$

similar to (13).

Besides Fig. 1, all the above discussion ignores the contribution of linear phase noise induced by the optical amplifier noise, i.e., the phase of $E_{0}+n_{1}+\cdots+n_{N}$. If the limitation of nonlinear phase noise is estimated based on the mothod of [1] in which the variances of linear and nonlinear phase noise are more or less the same, from Fig. 2, the mean nonlinear phase noise can be doubled. Because the mean nonlinear phase noise is proportional to the distance, the transmitted distance can be doubled if the nonlinear phase noise is the dominated impairment. Linear phase noise is correlated with neither nonlinear phase noise nor residual nonlinear phase noise. The variance of the overall phase noise, including both linear and nonlinear phase noise, is the sum of their corresponding variances. The $Q$-factor due to all phase noises, defined in [1], can be calculated accordingly.

Both Figs. 1 and 2 show that the linear compensator from [5-7], if optimized, is very close to the optimal MMSE compensator given by (4). Using (A.10) of Appendix A in both (7) and (8), the sub-optimal linear compensator in [7] can be derived as proportional to $(N+1) / 2+(N-1) \rho_{\mathrm{r}}^{2} / 2$.

As shown in Fig. 2, the variances of the residual nonlinear phase noise of the linear and optimal MMSE compensator have little difference. When linear and optimal MMSE compensators are used, the probability density functions of the residual nonlinear phase noise [9] may not be the same, especially the tail probabilities. With the approximation of (A.9) from Appendix A, the probability density function of the residual nonlinear phase noise is Gaussian distributed. In practice, the residual nonlinear phase noise has a larger tail probability than that of the Gaussian distribution [9]. While it is possible to derive the probability density function of the residual nonlinear phase for the linear compensator, that for nonlinear compensator is difficult to derive. 
This paper ignores the effects of fiber dispersion. If the nonlinear phase noise is defined as the phase noise induced by self-phase modulation of the signal and amplifier noise from an optical bandwidth matched to the signal, the signal and amplifier noise propagates in more or less the same speed. Induced by self-phase modulation, the nonlinear phase noise of (1) is not changed due to fiber dispersion. For high-order effects, the model of (1) needs to modify if fiber dispersion changes the optical waveform or optical pulse, reduces or increases the amplitude of $E_{0}$, and may change the effective length of $L_{\text {eff }}$. If dispersion compensation per span can reshape the optical waveform, to certain extend, fiber dispersion does not change the model of this paper.

\section{Conclusion}

When received intensity or amplitude is used to compensate for nonlinear phase noise, the optimal nonlinear MMSE compensator is derived analytically the first time. The MMSE compensator has a linear term proportional to the received intensity and a nonlinear term as the product of the received amplitude and the ratio of two modified Bessel functions of first kind. In term of the variance of the residual nonlinear phase noise, the linear compensator in which the correction term is proportional to the received intensity approximates the optimal MMSE compensator well.

\section{Appendix A. The estimation of $E\left\{\left|A+\xi_{1}\right|^{2}\left|A+\xi_{1}+\xi_{2}\right|\right\}$}

For a real value $A$ and two zero-mean complex circular Gaussian variables $\xi_{1}$ and $\xi_{2}$, we would like to find

$$
\varphi\left(\sigma_{1}, \sigma_{2}\right)=E\left\{\left|A+\xi_{1}\right|^{2} \mid \rho_{2}\right\}
$$

where $\rho_{2}=\left|A+\xi_{1}+\xi_{2}\right|, E\left\{\xi_{1}\right\}=E\left\{\xi_{2}\right\}=0, E\left\{\left|\xi_{1}\right|^{2}\right\}=2 \sigma_{1}^{2}$, and $E\left\{\left|\xi_{2}\right|^{2}\right\}=2 \sigma_{2}^{2}$. The estimation of (A.1) is used to derive the MMSE estimator of (4). The real value of $A$ can be used to represent the amplitude of $\left|E_{0}\right|$ that is a constant for both PSK and DPSK signal. Define $x_{1}+\mathrm{j} y_{1}=A+\xi_{1}$ and $x_{2}+\mathrm{j} y_{2}=A+\xi_{1}+\xi_{2}$, the joint probability density function (p.d.f.) of $x_{1}, y_{1}, x_{2}, y_{2}$ is

$$
f_{x y}\left(x_{1}, y_{1}, x_{2}, y_{2}\right)=\frac{1}{\left(2 \pi \sigma_{1} \sigma_{2}\right)^{2}} \exp \left[-\frac{\left(x_{1}-A\right)^{2}+y_{1}^{2}}{2 \sigma_{1}^{2}}\right] \exp \left[-\frac{\left(x_{2}-x_{1}\right)^{2}+\left(y_{2}-y_{1}\right)^{2}}{2 \sigma_{2}^{2}}\right] \text {. }
$$

Changing the variable to $x_{2}=\rho_{2} \cos \theta_{2}$ and $y_{2}=\rho_{2} \sin \theta_{2}$, the marginal p.d.f. of $\rho_{2}$ is a Rician distribution of

$$
\begin{aligned}
f_{\rho_{2}}\left(\rho_{2}\right) & =\int_{-\infty}^{+\infty} \int_{-\infty}^{+\infty} \int_{-\pi}^{+\pi} f_{x y}\left(x_{1}, y_{1}, \rho_{2} \cos \theta_{2}, \rho_{2} \sin \theta_{2}\right) \rho_{2} \mathrm{~d} \theta_{2} \mathrm{~d} x_{1} \mathrm{~d} y_{1} \\
& =\frac{\rho_{2}}{\left(\sigma_{1}^{2}+\sigma_{2}^{2}\right)^{1 / 2}} \exp \left(-\frac{A^{2}+\rho_{2}^{2}}{\sigma_{1}^{2}+\sigma_{2}^{2}}\right) I_{0}\left(\frac{A \rho_{2}}{\sigma_{1}^{2}+\sigma_{2}^{2}}\right),
\end{aligned}
$$

The estimation of (A.1) is

$$
\varphi\left(\sigma_{1}, \sigma_{2}\right)=\int_{-\infty}^{+\infty} \int_{-\infty}^{+\infty}\left(x_{1}^{2}+y_{1}^{2}\right) f_{x y \mid \rho_{2}}\left(x_{1}, y_{1} \mid \rho_{2}\right) \mathrm{d} x_{1} \mathrm{~d} y_{1}
$$

where the conditional p.d.f. is

$$
f_{x y \mid \rho_{2}}\left(x_{1}, y_{1} \mid \rho_{2}\right)=\frac{1}{f_{\rho_{2}}\left(\rho_{2}\right)} \int_{-\pi}^{+\pi} f_{x y}\left(x_{1}, y_{1}, \rho_{2} \cos \theta_{2}, \rho_{2} \sin \theta_{2}\right) \rho_{2} \mathrm{~d} \theta_{2} .
$$


The integration of (A.4) becomes

$$
\frac{\rho_{2}}{f_{\rho_{2}}\left(\rho_{2}\right)} \int_{-\pi}^{+\pi} \int_{-\infty}^{+\infty} \int_{-\infty}^{+\infty}\left(x_{1}^{2}+y_{1}^{2}\right) f_{x y}\left(x_{1}, y_{1}, \rho_{2} \cos \theta_{2}, \rho_{2} \sin \theta_{2}\right) \mathrm{d} x_{1} \mathrm{~d} y_{1} \mathrm{~d} \theta_{2} .
$$

Integrated first over $x_{1}$ and $x_{2}$, we get

$$
\begin{aligned}
& \frac{1}{2 \pi\left(\sigma_{1}^{2}+\sigma_{2}^{2}\right)^{2} I_{0}\left(\frac{A \rho_{2}}{\sigma_{1}^{2}+\sigma_{2}^{2}}\right)} \int_{-\pi}^{+\pi} \exp \left(-A \rho_{2} \cos \theta_{2} /\left(\sigma_{1}^{2}+\sigma_{2}^{2}\right)\right)\left[\sigma_{1}^{4}\left(\rho_{2}^{2}+2 \sigma_{2}^{2}\right)+\sigma_{2}^{4}\left(A^{2}+2 \sigma_{1}^{2}\right)\right. \\
& \left.+2 A \rho_{2} \sigma_{1}^{2} \sigma_{2}^{2} \cos \theta_{2}\right] \mathrm{d} \theta_{2} .
\end{aligned}
$$

The integration of (A.4) is

$$
\varphi\left(\sigma_{1}, \sigma_{2}\right)=\frac{\sigma_{1}^{4}\left(\rho_{2}^{2}+2 \sigma_{2}^{2}\right)+\sigma_{2}^{4}\left(A^{2}+2 \sigma_{1}^{2}\right)}{\left(\sigma_{1}^{2}+\sigma_{2}^{2}\right)^{2}}+\frac{2 A \rho_{2} \sigma_{1}^{2} \sigma_{2}^{2}}{\left(\sigma_{1}^{2}+\sigma_{2}^{2}\right)^{2}} \frac{I_{1}\left(A \rho_{2} /\left(\sigma_{1}^{2}+\sigma_{2}^{2}\right)\right)}{I_{0}\left(A \rho_{2} /\left(\sigma_{1}^{2}+\sigma_{2}^{2}\right)\right)} .
$$

For the case of high optical SNR, the expectation of (A.1) can be approximated by

$$
\varphi\left(\sigma_{1}, \sigma_{2}\right)=E\left\{\left|A+\xi_{1}\right|^{2}|| A+\xi_{1}+\left.\xi_{2}\right|^{2}\right\} \approx E\left\{A^{2}+2 A \mathfrak{R}\left(\xi_{1}\right) \mid A^{2}+2 A \mathfrak{R}\left(\xi_{1}+\xi_{2}\right)\right\},
$$

where $\mathfrak{R}(\cdot)$ denotes the real part of a complex number. Using the approximation of (A.9), the expectation of (A.1) becomes

$$
\varphi\left(\sigma_{1}, \sigma_{2}\right) \approx \frac{\sigma_{2}^{2}}{\sigma_{1}^{2}+\sigma_{2}^{2}} A^{2}+\frac{\sigma_{1}^{2}}{\sigma_{1}^{2}+\sigma_{2}^{2}} \rho_{2}^{2} .
$$

\section{References}

[1] J.P. Gordon, L.F. Mollenauer, Opt. Lett. 15 (1990) 1351.

[2] A.H. Gnauck, et al., in: Proc. OFC'02, Optical Society of America, Washington, DC, 2002 (postdeadline paper FC2).

[3] R.A. Griffin, et al., in: Proc. OFC'02, Optical Society of America, Washington, DC, 2002 (postdeadline paper FD6).

[4] B. Zhu, et al., in: Proc. ECOC'03, COM Center, Denmark, 2002 (postdeadline paper PD4.2).

[5] X. Liu, X. Wei, R.E. Slusher, C.J. McKinstrie, Opt. Lett. 27 (2002) 1616.

[6] C. Xu, X. Liu, Opt. Lett. 27 (2002) 1619.

[7] K.-P. Ho, J.M. Kahn, Available from <http://arXiv.org/physics/0211097>.

[8] S. Norimatsu, K. Iwashita, K. Noguchi, IEEE Photon. Technol. Lett. 4 (1992) 765.

[9] K.-P. Ho, to be published in J. Opt. Soc. Amer. B, available from http://arXiv.org/physics/0301018. 